\title{
A New MAC Protocol for Moving Target in Distributed Wireless Sensor Networks
}

\author{
Jijun Zhao, Xiang Sun, Zhongcheng Wei, Zhihua Li \\ School of Information \& Electronic Engineering, Hebei University of Engineering, Handan, China \\ E-mail:sx198574@sohu.com \\ Received November 29, 2010; revised December 16, 2010; accepted February 21, 2011
}

\begin{abstract}
A wireless sensor network (WSN) is composed of many nodes with limited power supply; most nodes are stationary in the network which could probably involve a few mobile nodes. Various medium access control (MAC) protocols specially aimed at a target locating application for WSNs have been proposed. However, most of these protocols based on the problem of energy-limited does not consider the mobility of nodes. Therefore, in order to solve such problem, this paper proposes a MAC protocol-Distribute Moving-MAC (DM-MAC). Under the condition of keeping high network coverage, the protocol utilizes the redundancy of nodes to strengthen the robustness and energy efficiency of network, and decreases the packet loss rate of the mobile node's communication links for realizing reliable communication of two nodes. Simulation indicates that the new protocol has higher energy efficiency, lower packet loss rate and higher network coverage which suit for wireless sensor network with mobile nodes well.
\end{abstract}

Keywords: Wireless Sensor Network (WSN), Distribute Moving-MAC (DM-MAC), LDAS, EAR, Mobility

\section{Introduction}

A wireless sensor network (WSN) has characteristics of self-organization, multi-hop, large scale of monitoring and energy constraints. Recently, many research institutions have studied on this area and have a great breakthrough on it. Because WSN has a strong ability of processing ability within network, it has been applied to various situations, such as battlefield monitoring, machine fault monitoring, biological monitoring, intelligent furniture and so on. MAC protocol in WSN is mainly to realize the reliable communication of point-to-point (node to node), sleeping mechanism, synchronization mechanism and so on. Therefore, MAC protocol has a decisive function to whole network due to a great influence on energy efficiency, scalable to node density, frame synchronization, fairness, bandwidth utilization, flow control, and error control for data communication.

The most key feature in WSN is the limitation of node energy, therefore, most MAC protocols have focused on the energy saving in WSN without considering the mobility of nodes. Nevertheless, there have been introduced a certain amount of mobile node in many position systems and environmental monitoring systems. Thus, increasing the reliability of the mobile nodes communica- tion under the condition of ensuring network connection and coverage becomes a mayor research trend of MAC protocol study. In recent researches, Traffic-Adaptive Medium Access protocol (TRAMA) [1] and WiseMAC protocol [2] solve node sleeping mechanism well and limit energy consumption of the node in a certain low value, but bring about a large packet loss rate after several mobile nodes being added into network. The Lightweight Deployment-Aware Scheduling (LDAS) algorithm proposed in [3] solves the problem of low coverage (including sensor coverage and communication coverage) which caused by nodes' sleeping mechanism; however, it doesn't have a mechanism to realize the reliable communication, so it doesn't accomplish dynamic communication between the different nodes. The Eavesdrop-And- Register (EAR) algorithm proposed in [4] which based on the Self-organizing Medium Access Control for Sensor networks (SMACS) algorithm can realize the reliable communication between mobile node and fixed node by depending on increasing the control head packets, but when adding the number of mobile nodes, it results in the packets collision with a high probability rate that leads to the node energy consumption much faster and it also doesn't guarantee high rate of coverage in the monitoring area. The mobility-aware 
MAC protocol for sensor networks (MS-MAC) proposed in [5] can adjust the scheduling mechanism dynamically acco-rding to velocity of the node, but this protocol is based on the Senor-MAC (SMAC) protocol in [6,7], which doesn't consider the problem of reliable communication of mobile node and the network coverage. Here the network refers to this paper is the areas that are collectively covered by the set of all sensors.

Based on the above researches, we find that the existing MAC protocols are almost proposed based on the problems of energy consumption, network coverage and network connection. In the recent researches, very few MAC protocols could be used in a mobile network. Although a few protocols could support a number of the mobile nodes, they also exist the problem of high energy consumption, limit number of mobile nodes and low network coverage, etc [8-14]. DM-MAC proposed in the paper is based on the network environment which exist many stationary nodes and several mobile nodes. The protocol decreases occurrence probability of blind hole and solves unreliable point-to-point communications which are caused by LDAS algorithm, and improves the communication mode in EAR algorithm and intelligent scheduling mechanism in MS-MAC protocol to realize the communication between mobile nodes and stationary nodes. Meanwhile, the protocol could ensure the network having a good performance in network coverage and network connection which also enhance robustness of the network.

In the network, the function of stationary node is to collect and monitor sensor data of surrounding environment, locate the geographical position of the mobile nodes and send collecting data to sink node. As the target node need to be located, mobile node is to broadcast signals for the purpose of location by stationary node; meanwhile, mobile nodes also collect environmental data and send them to sink node by the stationary. Generally, mobile node is always composed of stationary node which fixed on the movable objects. We consider that the limitation of energy and hardware is not a key problem of the mobility, so some management tasks could be given to mobile nodes for achievement.

The paper is organized as follows. In Section 2, we analysis the existing protocols which adapt to the distribute network for moving target; while Section 3 proposes a new protocol whose performance is superior to the other existing protocols. Simulation results are presented in Section 4 and Section 5 concludes our work.

\section{Related Protocols Analysis}

According to the aspects of sleeping mechanism, network coverage and building communication link, etc, we mainly analyze the following three protocols, with comparing their advantages and disadvantages for the future research.

\subsection{EAR Algorithm}

The basic idea of EAR is to build reliable communication link though adding handshaking times and using SNR value of receiving signals. In the EAR algorithm, links are build up between mobile nodes and stationary nodes considering four kinds of frames: Broadcast Invite (BI), Mobile Invite (MI), Mobile Response (MR) and Mobile Disconnect (MD).

The detail realization process of the algorithm is shown in Figure 1. BI sent by stationary node is used to invite mobile node to join in the communication. Moreover, stationary node would last this action until its registration form is full. When registration form is full, compare SNR value of the stationary node to the value of other nodes which have already connected with mobile nodes, write the higher value into the form. If BI frame has existed in the registration form, update registration form according to the time field in BI frame. MI frame sent by mobile nodes is to response to BI frame and request to build up a connection. MR frame is to decide whether stationary node accepts the MI frame. When communication link can be established, the stationary node will send MR frame to mobile node in acknowledgement of accepting the connection request. MD frame is to disconnect the link. After connecting with the stationary node, mobile node continues to listen to this stationary node. When the SNR value of signal which mobile node receives is lower than the threshold we set before or there are more appropriate stationary nodes communicating to the mobility, the mobile node sends MD frame to inform stationary node to interrupt linkage. After stationary node receives this frame, it deletes information of mobile node in the registration form.

The disadvantage of EAR algorithm: at first, it could only be used in the WSN based on TDMA technology; meanwhile, the network topology could only be cluster so that the algorithm could ensure the low time delay in network. Second, energy efficiency of stationary node is low, because stationary node needs to send extra BI

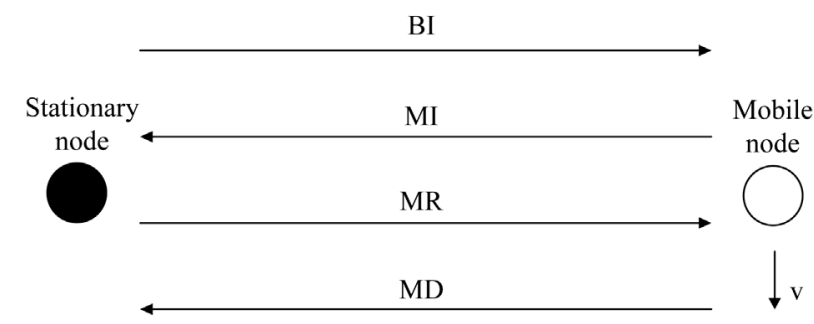

Figure 1. The communication process of EAR algorithm. 
frame periodically to find out mobile node. Third, the algorithm doesn't propose a suitable sleeping mechanism based on high redundant network, so it leads to large number of collision in the high density sensor network which could result in decreasing the energy efficiency of node, shorting the life of network and prolonging the communication latency.

\subsection{MS-MAC Algorithm}

MS-MAC algorithm adjusts node sleeping time dynamically according to the velocity of mobile node. Its main idea is based on SMAC protocol. The advantage of the algorithm is that it proposes a kind of sleeping mechanism which suits for the mobile networks. Similar with SMAC, MS-MAC algorithm sends SYNC frame to build up virtual clusters in the period of networking. In every virtual cluster, all the nodes keep synchronous and the nodes on the edge of two virtual clusters should follow synchronous periods of the two virtual clusters. Therefore, at the beginning of every scheduling mechanism, nodes send SYNC to build up virtual cluster to keep time synchronous. When the mobile nodes join in the network, it must follow time synchronous mechanism of the virtual cluster. So when the mobile node moves from one virtual cluster to another, it must wait until the next scheduling mechanism begins, therefore it could know the time synchronous mechanism of another virtual cluster. Generally, this mechanism increases time delay in communication. For example, suppose that one scheduling cycle is 2 minutes and nodes stay at wake-up status in 10 seconds in every 2 minutes, so mobile node at most needs to wait 1 minute 50 seconds to know synchronous mechanism of the virtual cluster. MS-MAC algorithm decreases the time delay by adjusting scheduling mechanism dynamically for adapting the velocity of the mobility. At first, set a value $v_{0}$ if velocity of mobile node is higher than $1 / 4 v_{0}$, make scheduling cycle as 1 minute if the velocity of mobile node is higher than $1 / 2$ $v_{0}$, then make scheduling cycle as 30 seconds, etc.

Though MS-MAC algorithm could use the velocity to adjust scheduling cycle dynamically, it can't ensure the reliable communication between stationary nodes and mobile nodes; meanwhile, this kind of sleeping mechanism also doesn't ensure the high rate of coverage and connection of the whole network.

\subsection{LDAS Algorithm}

When the node doesn't know its own coordinate position information and the angle information of its neighbors, the sleeping mechanism can use the number of node's neighbors to decide whether go to sleep, (the node's neighbors defined in the paper are all the nodes in the circular area whose radius is the node sensing range $r$ ), so that it can cover the most sensing area on the base of high energy efficiency. The probability formula that the sleeping node is fully covered by its neighbors is as follows [3]:

$$
1-n 0.609^{n-1} \leq \operatorname{Pr}(A) \leq 1-n 0.609^{n-1}+\xi
$$

$\xi=0.5 * n *(n-1) *(0.276)^{\mathrm{n}-1} . n$ is the number of the awaken node's neighbors ( $n$ could be selected according to QoS requirement). In the paper, we suppose that communication radius is equal to sensing radius. So when $n=$ 3 , the probability that node can be fully covered is: $0 \leq$ $\operatorname{Pr}(\mathrm{A}) \leq 0.113$, when $n=5$, then the probability that node can be fully covered is: $0.312 \leq \operatorname{Pr}(\mathrm{A}) \leq 0.370$. Suppose that $n=3$ could satisfy the QoS requirement, meanwhile, the node finds three more neighbors which are not in sleeping status, the node considers its sensing rang being covered by its neighbors and the node could go to sleeping status. In this case, it could happen in following situations, as Figure (a) shows, the node $O_{1}$ could be aware of its three neighbors $\mathrm{O}_{2}, \mathrm{O}_{3}, \mathrm{O}_{4}$, so it goes to sleeping status. But when mobile node goes though the shadow region, any nodes in the network could not be aware of location of mobile node until the node $O_{1}$ ends up its sleeping status. Now we consider the situation that the smallest sensing area which could be covered by its neighbors, as Figure (b) shows, suppose that the sensing radius and communication radius are R. Because central angle is $120^{\circ}$, the whole area of shadow region is as follows:

$$
S=\frac{2}{3} \pi R^{2}-\frac{\sqrt{3}}{4} R^{2}
$$

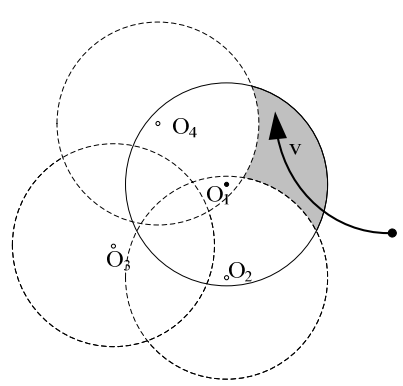

(a)

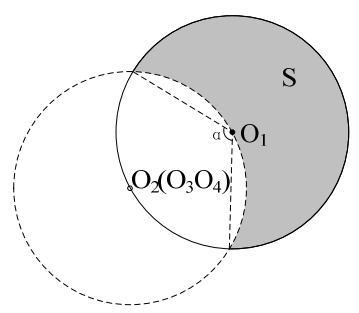

(b) 
Because when $n=3$, the probability which the node's sensing area/communication area fully covered by its neighbor is among [0, 0.113]. Suppose that $\mathrm{R}=10 \mathrm{~m}$, so we can calculate the area $S \approx 166.032 \mathrm{~m}^{2}$; when $n=5, S \approx$ $130.833 \mathrm{~m}^{2}$. Therefore, from the above data, it is known that if only using LDAS sleeping algorithm, there will be a large number of blind holes in the network, which could make mobile node disappear.

Although a bigger blind hole occurs in network with a certain probability, LDAS has its own advantages: 1) the algorithm is simple that adapts to a large scale network, the nodes in the network can be random distributed;2) the algorithm needn't know geographical position information of neighbor nodes; 3) the algorithm can adjust dynamically to achieve the requirement of QoS; 4) the algorithm has a strong resistant ability for channel error and collision; 5), the blind hole appears randomly and has a small influence on event monitoring relatively; 6) the algorithm needn't keep time synchronous mechanism. However, LDSA isn't the mechanism which could realize a reliable point-to-point communication, so it makes the communication between mobile node and stationary node unrealized.

\section{Design of the DM-MAC Protocol}

Combining with the above three kinds of algorithms, we design a MAC protocol based on distributed networkDM-MAC. On the premise of ensuring the network coverage, this protocol could realize the reliable communication between stationary node and mobile node. Meanwhile, the protocol uses the intelligent scheduling mechanism proposed in MS-MAC algorithm to decrease the delay in the communication and to decrease the disappearance probability of mobile node which caused by the sleeping mechanism.

\subsection{Main Design of the Protocol}

\subsubsection{Network Initialization}

Suppose that nodes in the network are distributed randomly and sensing radius of every node $r$ is equal to its communication radius $R$. Because the energy of mobile node can be considered infinitely, so we can increase the communication radius of the mobile node to $2 R$ by using power control technology. The initial stage of establishing network, LDSA algorithm will be used to initialize the sleeping mechanism of every node. The concrete process is as follows: At first, the stationary node checks the number of its working neighbors (the number of working nodes in the sensing range of the node) in every CT (Checking Time) time. Second, the stationary node broadcasts its beacon message to show it existing in the network. The beacon message packet includes two parts: the nodes' ID and the number of working neighbors. When other nodes receive this beacon message, update related node's information in the receivers. If the node doesn't receive the beacon message send by its neighbors in a long period, it will make its neighbors turn into sleeping status. So every node has a neighborhood table. According to requirement of QoS, we can estimate the required number of working nodes. If the number of existing working neighbors is more than required, the node sends ticket packets to its working neighbors. In working stage, if the node has enough tickets to turn into sleeping status, it should also check the number of the working neighbors again to eliminate the nodes that sleep at the same time. So if the number of tickets node received is more than TT (Ticket Threshold), the node turns into ready-to-off status, and then the node goes to backoff stage (the backoff time is: $\operatorname{random}\left(0, W_{\text {max }}\right)$ ). Next, the node checks for the second time that whether it has enough working neighbors to make itself turn into sleeping status, if condition is satisfied, the node turns into sleeping status(off-duty) until passing by a time segment of ST (Sleep Time). After sleeping a period of ST, the node goes into on-duty status and clears the values of tickets.

\subsubsection{Communication with Stationary Node and Mobile Node}

When mobile node goes into the network, it broadcasts $B I$ signal periodically at first (In EAR algorithm, $B I$ is sent by stationary node which is used to invite the mobile node to join the communication. In order to decrease the energy consumption of stationary node and the probability of collision, we make mobile nodes to send $B I$ signal). $B I$ frame includes the velocity of mobile node, the ID of mobile node and several sensing parameters collected by mobile node. After the velocity of mobile node (the velocity value can be gained by RF Doppler Shifts [15]) is received by stationary node, it would be compared with VT (Velocity Threshold) which is set before the stage of establishing network, if the two values are different; changing the $S T$ value defined in the sleeping mechanism to adjust the node's sleeping time. In order to make the stationary node adjust its scheduling mechanism in advance, we apply power control technology to increase the mobile node's transmit power for enlarging its communication radius. After setting proper scheduling mechanism, mobile node sends $B I$ frame continuously and periodically, when stationary node receives $B I$ frame, it sends $M I$ frame to the mobility to ensure that it has received the $B I$ frame successfully (in EAR algorithm, $M I$ frame is sent by mobile node). In the $M I$ frame, it includes the ID of stationary node and the SNR value of 
the $B I$ that is received by stationary node. After the mobile node receives $M I$ frame, it accords to SNR value in the connected communication links to decide whether to establish the connection, if the SNR value is bigger than the existing SNR value in the communication links, then the mobile node interrupts the connected link, establishes the link to the existing stationary node and then sends $M R$ frame to establish the connection. If the SNR value is smaller than the existing SNR value in the communication links, the mobile node neglects the $M I$ frame and maintains the existing communication link. In order to decrease the times of comparison, we set $E_{0}$ as threshold of SNR in advance, when SNR value in $M I$ frame is smaller than $E_{0}$, the mobile node neglects $M I$ frame directly. When SNR value in the connected communication link is smaller than $E_{0}$ or smaller than any SNR values in the established communication links, mobile node must send $M D$ to the stationary nodes to interrupt the communication link. Notice that, after stationary nodes have established the communication with the mobile nodes, even if stationary nodes maybe satisfy the condition of turning into sleeping status, they can't go to sleep until disconnecting the communication with the mobile nodes.

\subsection{The Analysis of DM-MAC Protocol in Process and Time Sequence}

The content of DM-MAC protocol realized in the stationary node and mobile node are not the same, stationary node mainly implements sleeping mechanism, intelligent scheduling mechanism and reliable point-to-point communication mechanism, meanwhile, the function of mobile nodes is relative simple, it mainly completes reliable point-to-point communication mechanism and maintains SNR table in dynamic neighbor nodes. Here we focus on the protocol process of stationary nodes in detail. The basic flow chart is as Figure 2 shows. We suppose that when nodes send packets, the channel is free. The communication of stationary node includes the communication between stationary node and mobile node, and the communication between stationary nodes. Figure 2 shows the communication basic flow on stationary node and Figure 3 shows the communication basic flow on mobile node, we apply CSMA/CA mechanism and $\mathrm{S}-\mathrm{MAC}$ to realize the communication between the stationary nodes so that the data which stationary nodes collect from the surrounding environment and the mobility information which stationary nodes collect can be successfully multi-hop to the sink node.

Some nodes are selected to analyze their time sequence. The topology distribution map is shown in Figure 4. A, $\mathrm{B}, \mathrm{C}$ and $\mathrm{D}$ are stationary nodes, $\mathrm{M}$ is a mobile node, the sensing area of node B is fully covered by node A, C and D. Meanwhile, suppose that node B is also fully covered by its neighbors (In order to describe conveniently, we don't consider the case of neighbors of node A, C and D temporarily). We suppose that when $n=3$ can satisfy the QoS requirement, so if the number of working neighbors is 3 , then node $\mathrm{B}$ could go to sleep. When the mobile node moves into the network, suppose that its communication radius is $r_{1}=2 R$ (which is two times bigger than stationary node's communication radius), therefore, if node $\mathrm{M}$ moves as Figure 4 shown, node A will listen to $B I$ frame at first which is sent by $\mathrm{M}$ periodically, meanwhile, A adjusts the $S T$ value dynamically by receiving the velocity in $B I$ frame. According to the SNR in MI frame, when $\mathrm{M}$ continues moving, it will receive more MI signal, the node will choose the best nodes to establish communication link (We can set how many number of stationary nodes should be linked with the mobile nodes beforehand). The detail time-slot chart of establishing the link between stationary nodes and mobile nodes is as Figure 5 shows. We suppose that every mobile node can build communication link with only one stationary node.

\subsection{The Implementation of DM-MAC Protocol}

In the design of the whole DM-MAC protocol, we mainly implement the reliable point-to-point communication mechanism between stationary nodes and mobile nodes with intelligent sleeping mechanism. The sleeping mechanism proposed in LDAS algorithm will appear a large area of blind holes because communication area of node having been on off-duty status is not fully covered by its neighbors. The sleeping mechanism proposed in LDAS algorithm will appear a large area of blind holes because communication area of node having been on off-duty status is not fully covered by its neighbors. Thus in DM-MAC protocol, we enlarge the mobile nodes' communication radius to make sleeping node foresee the situation of the mobile nodes in early time, so the nodes can adjust its own scheduling mechanism after obtaining the velocity of mobility. The parameters of protocol we need to set beforehand are shown in Table 1.

The fames involve in the DM-MAC protocol are as follows: neighborhood table packet, beacon message frame, ticket frame, $B I$ frame, $M I$ frame, $M R$ frame and $M D$ frame, their concrete frame structures are shows from Figure 5 to Figure 11. Since the new protocol is aimed to the MAC layer, when designing the frame, we don't add packaging of physical layer and higher layer in the frame, such as packaged frame length in physical layer.

In order to realize the sleeping mechanism which could 


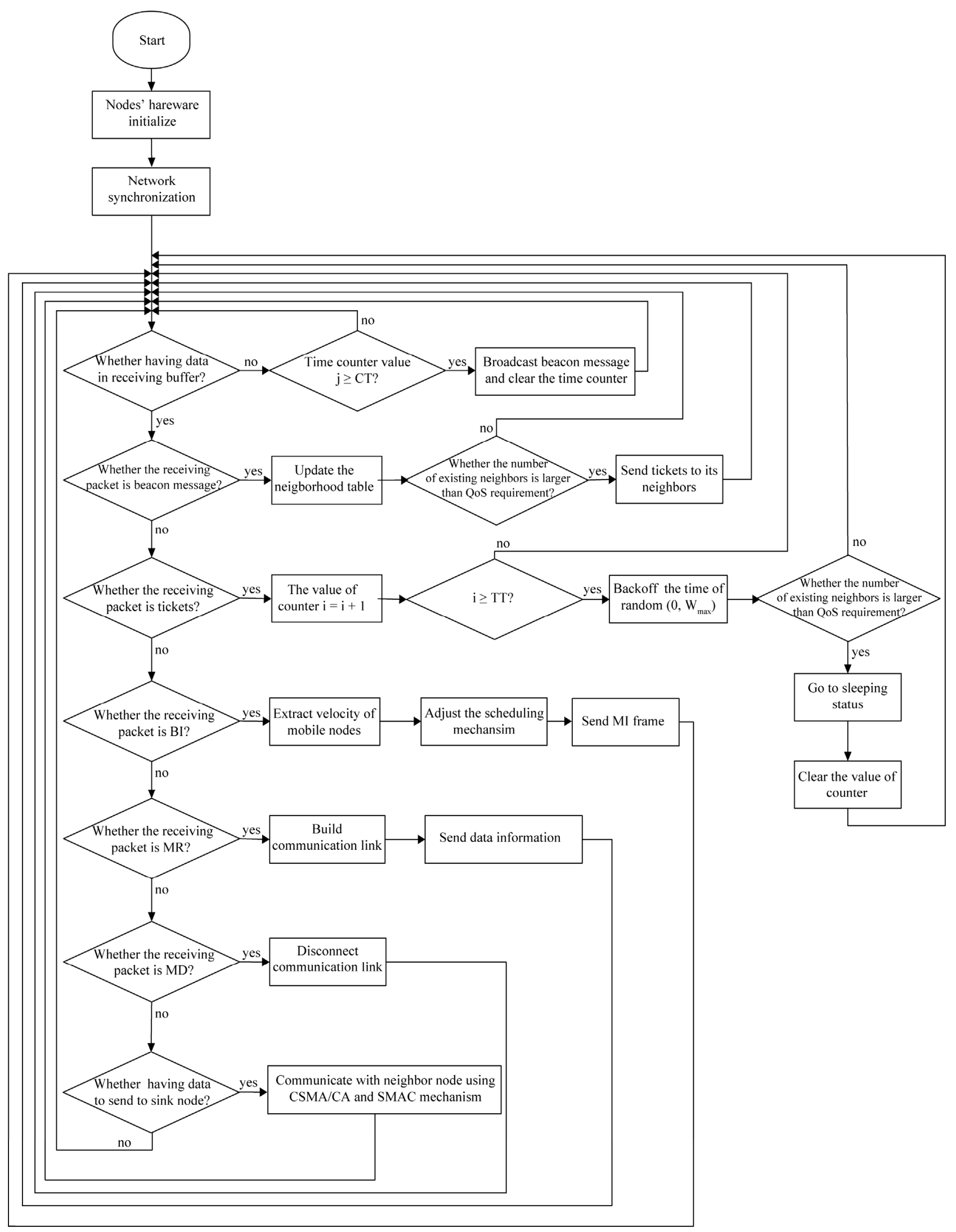

Figure 2. The protocol flow chart of stationary node. 


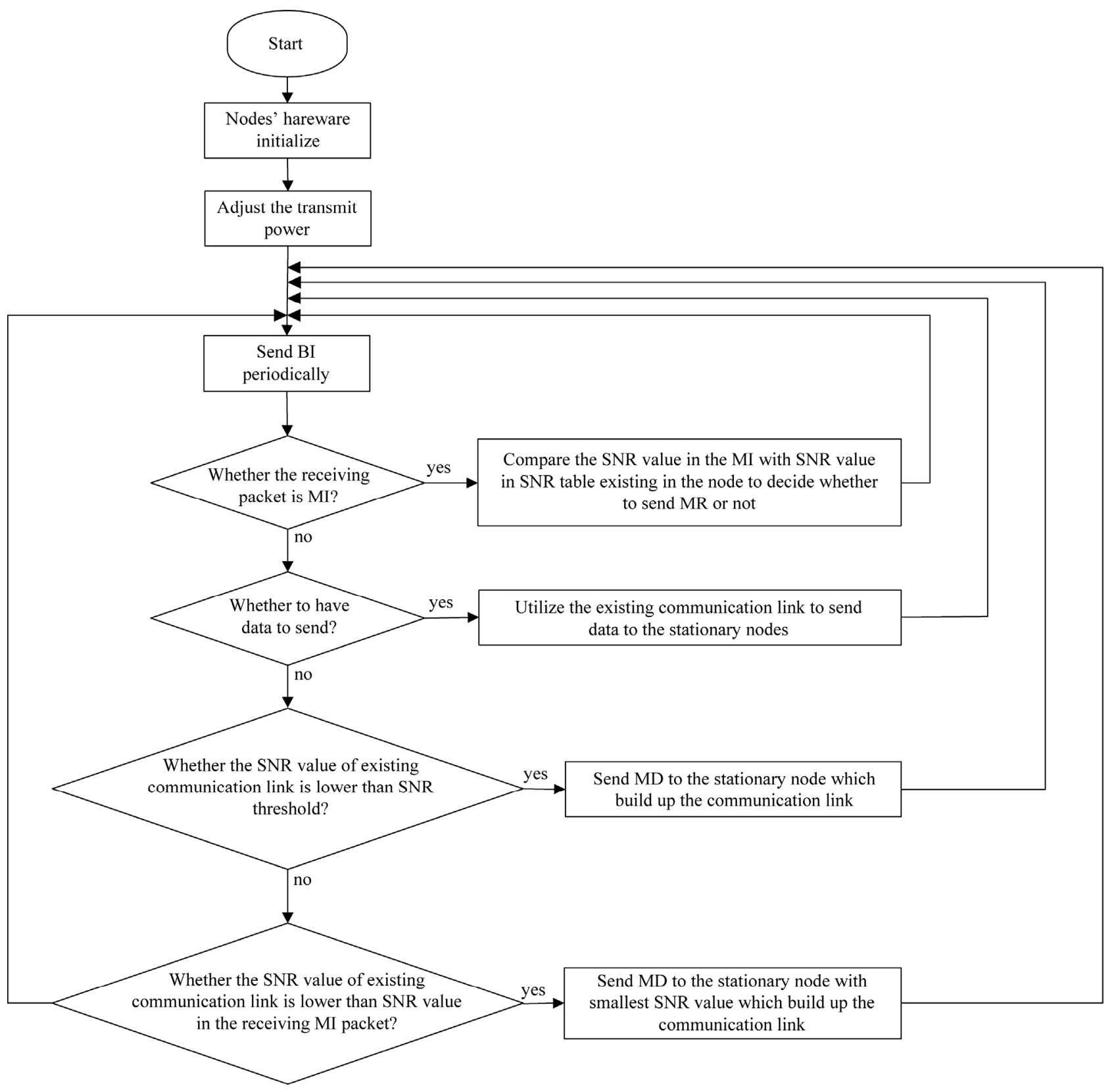

Figure 3. The protocol flow chart of mobile node.

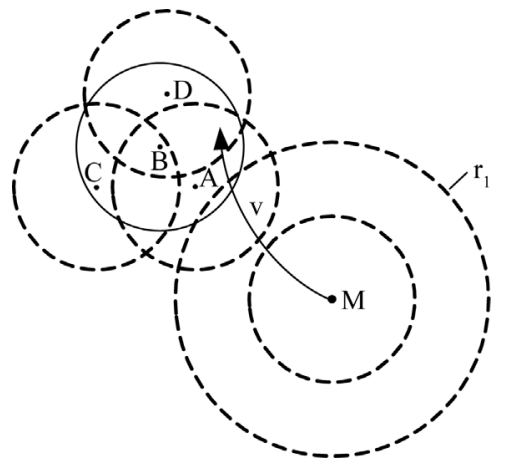

Figure 4. The topology chart of the nodes. ensure high sensing coverage, every node must maintain a sheet of neighborhood table. The detail table heading is followed:

ID is the ID number of the neighbors, Mode is the current status of the node, NoN is the number of the neighbors and Update Time is the time when neighborhood updates its content. Meanwhile, in order to compare the SNR value of receiving signal, mobile node also need maintain a sheet of SNR table. Its table heading is as follow:

ID is the ID number of transmitting node and SNR value is the signal-to-noise ratio of receiving signal. 
Table 1. The input parameters list of MAC layer.

Ticket Threshold (TT)

Sleeping Time (ST)

Neighborhood Table Checking (CT)

Beacon Message Interval Time (BT)

Maximum Back-off Time

Velocity Threshold (VT)

SNR Threshold
The threshold of the number of tickets node has been received which is to judge the node whether to go to sleep. It relates to the QoS requirement.

The sleeping time of the stationary nodes.

The time interval of updating the nodes' information in the neighborhood table.

The time interval of node sends beacon message.

The maximum value of back-off time: $\mathrm{W}_{\max }$

The threshold is to compare with the velocity of mobile node which is to adjust the sleeping time of the node dynamically.

The threshold is to compare the SNR value of received signal which is to decide whether to build the communication link or not

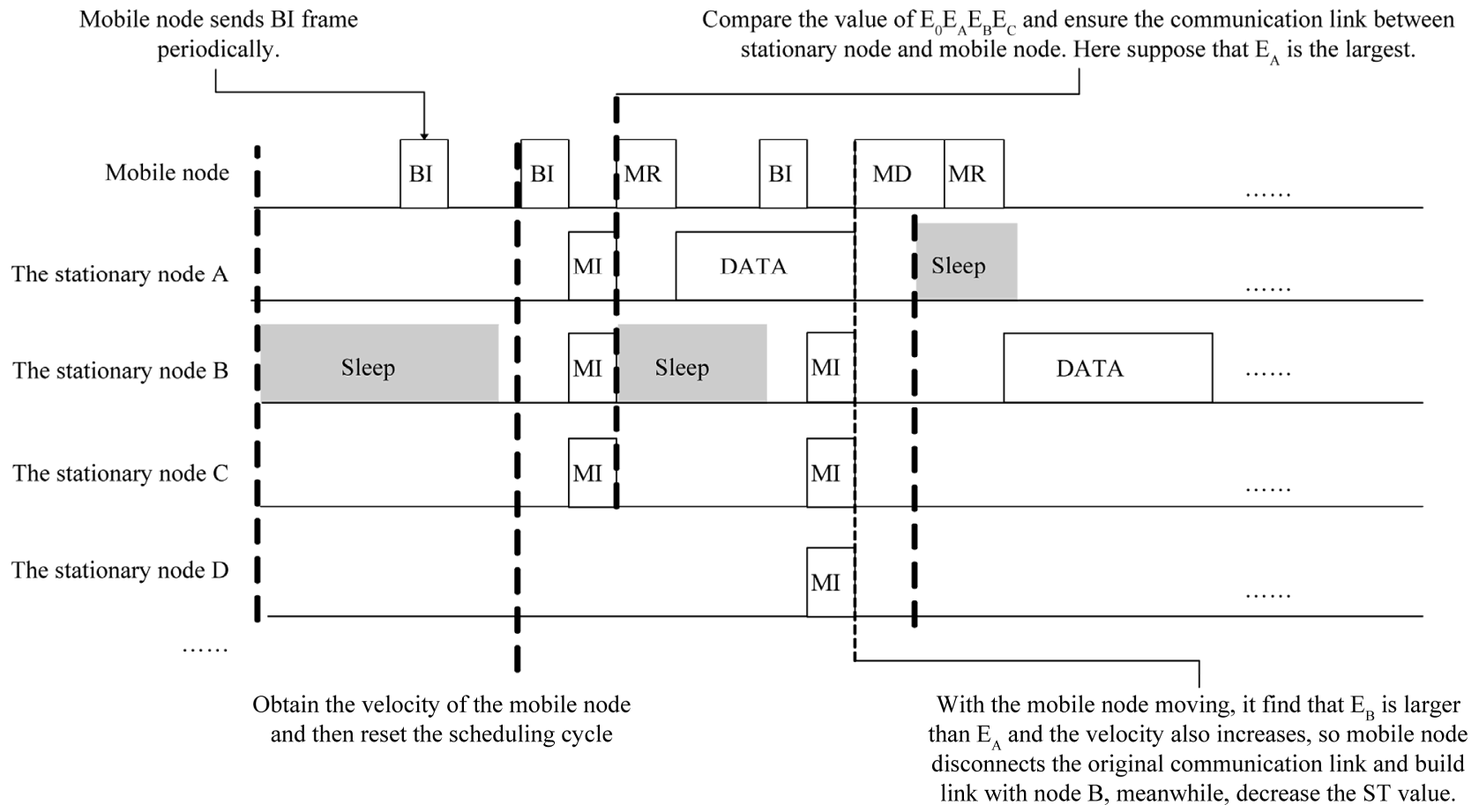

Figure 5. The time-slot chart of DM-MAC protocol.

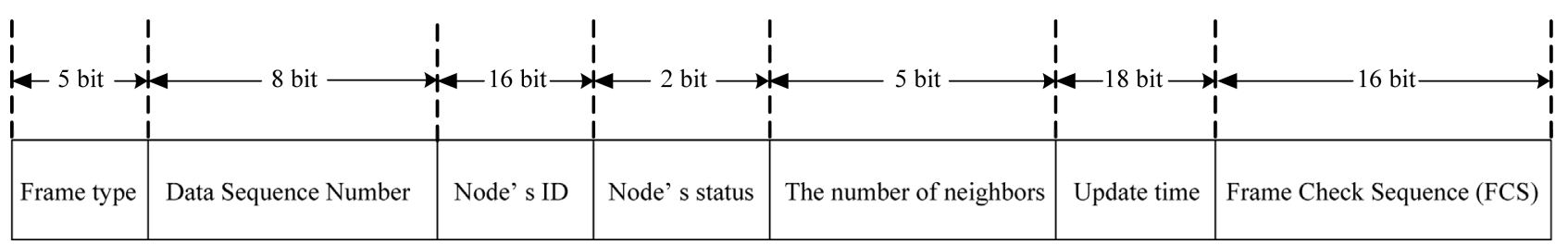

Figure 6. The packet structure of neighborhood table.

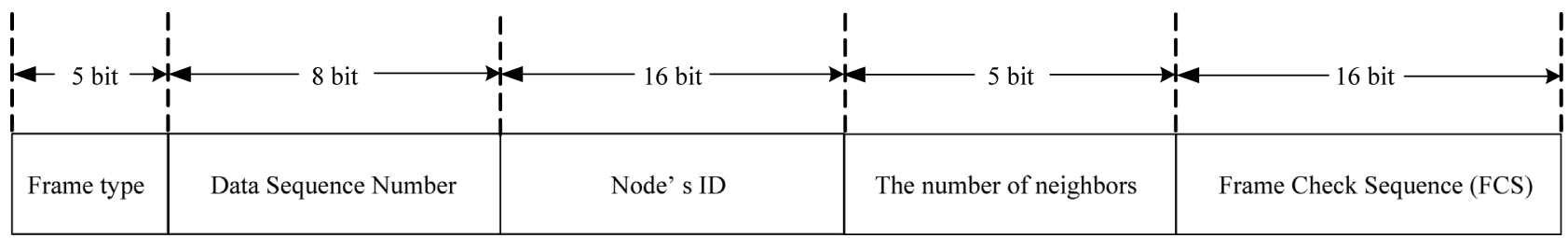

Figure 7. The packet structure of beacon message. 


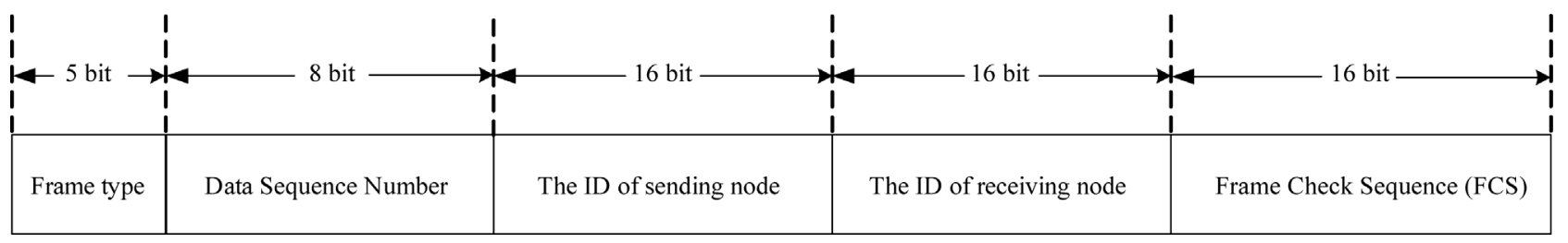

Figure 8. The packet structure of ticket.

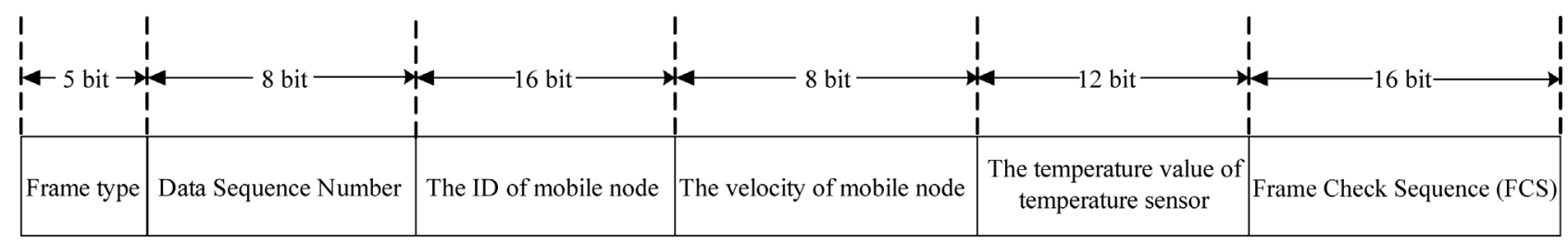

Figure 9. The packet structure of BI.

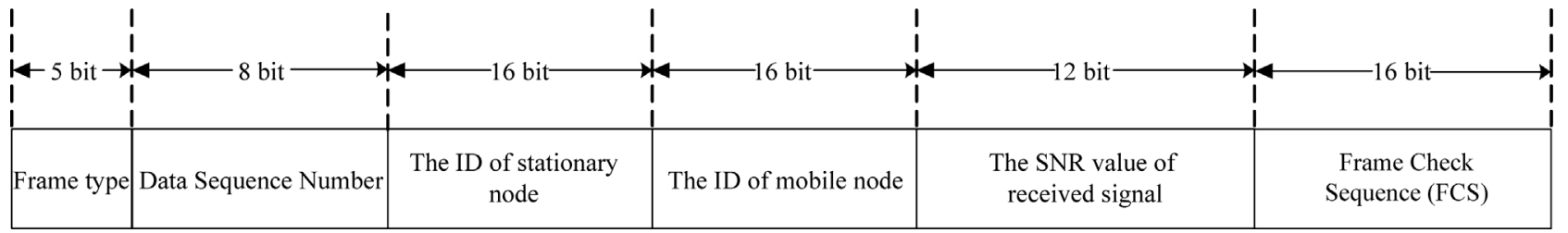

Figure 10. The packet structure of MI.

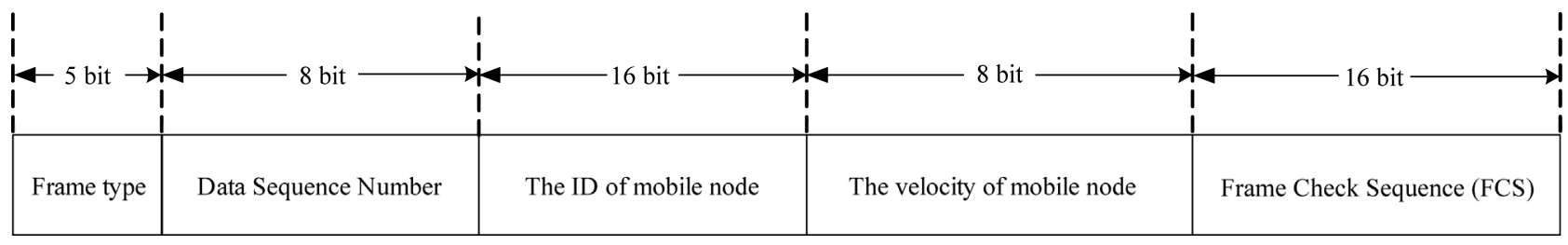

Figure 11. The packet structure of MR and MD.

Table 2. The table heading of neighborhood table.

\begin{tabular}{|c|c|c|c|}
\hline ID & Mode & NoN & Update Time (hour:minute:second) \\
\hline
\end{tabular}

Table 3. The heading of SNR table.

ID

SNR value

\section{Simulation Results}

In order to analyze the performance of DM-MAC protocol, we simulate the protocol in PC. Suppose that energy consumption ratio of transmitting, receiving (idling) and sleeping is 10:4:0.01. There are 200 stationary nodes randomly distributed in an area of $150 \times 150 \mathrm{~m}^{2}$, sensing radius and communication radius of the stationary nodes are $20 \mathrm{~m}$, communication radius of mobile node is $20 \mathrm{~m}$. Figures 12 and 13 show the case of node distribute and node sensing/communication coverage. We set $S T=1$ $\min , C T=10 \mathrm{sec}, B T=15 \mathrm{sec}, V T=3 \mathrm{~m} / \mathrm{s}, T T=8$, the communication channel is $2405 \mathrm{MHz}$, when $n=3$, QoS requirement could be satisfied.

\subsection{Connection Rate of Mobile Nodes}

Under the above condition, comparing with DM-MAC and LDAS + EAR, we simulate the connection rate of mobile nodes in the network (the connection rate of mobile nodes $=$ the number of mobile nodes which have already connected with stationary node/the number of whole mobile nodes). Suppose that there are 200 stationary nodes randomly distributed in the area, the simulate results are shows in Figure 14. In the figure, we know that when the number of mobile node is few, LDAS + EAR protocol has higher reliability of communication, but when the number of mobile node increases, packet loss rate relatively increases and the connection rate of mobile nodes decreases obviously. When mobile nodes are added up to 100 , nearly $40 \%$ mobile nodes disappear. This is because LDAS + EAR protocol may bring in big blind holes. Of course collision also brings in disappearing which is the main factor either when the mobile 


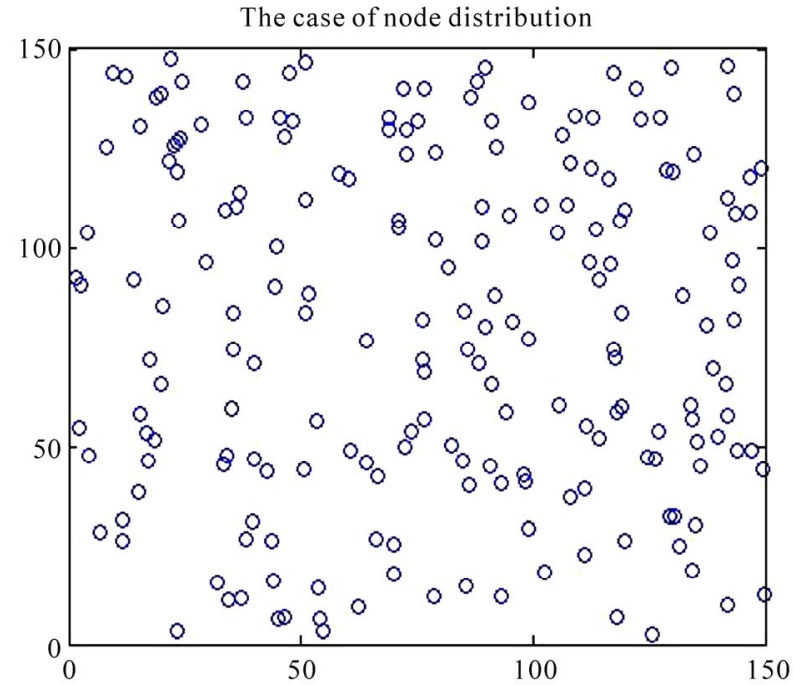

Figure 12. Node distribution.

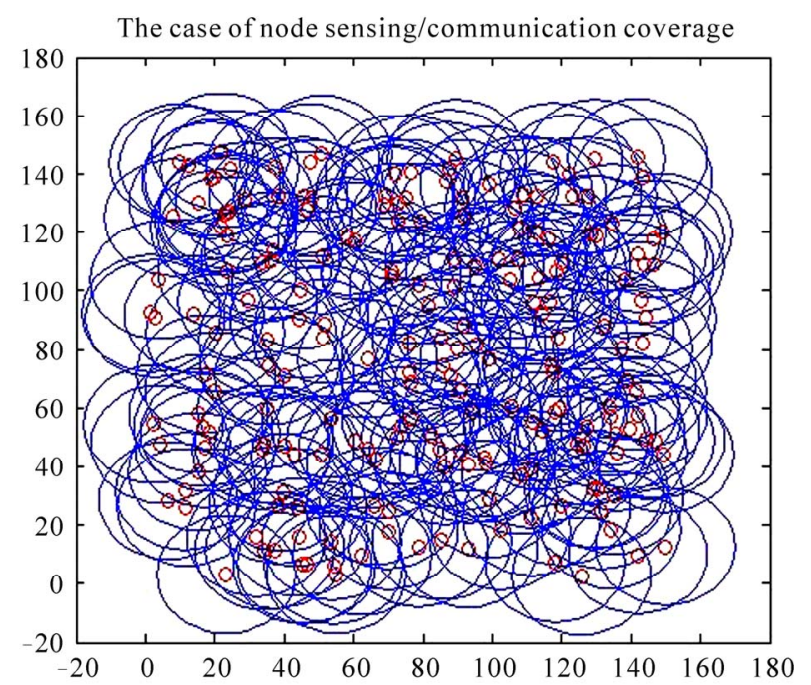

Figure 13. Node sensing/communication coverage.

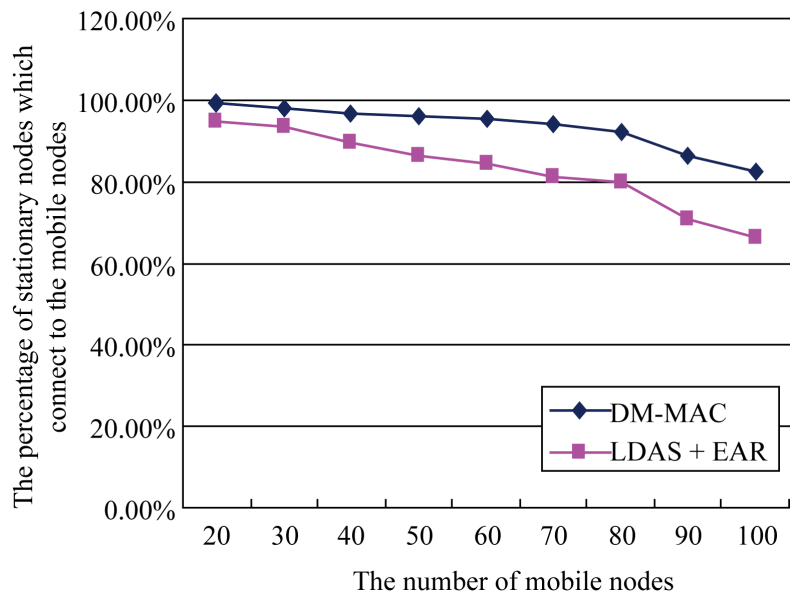

Figure 14. The connection rate of mobile nodes. nodes add, but in the paper we don't consider this factor of decreasing connection rate. Although the blind holes randomly appear, when the number of mobile nodes increases, the probability mobiles appears in the blind holes will also increases, thus the phenomenon that mobile nodes "disappear" will increase. The other curve in the figure is the mobile connection rate trend of DM$\mathrm{MAC}$, though the connection rate of mobile nodes also decreases with increasing the number of mobile nodes, its decreasing rate is obvious slower compared to the LDAS + EAR protocol. That is because DM-MAC protocol uses mobile nodes to broadcast BI frame periodically and stationary nodes to foresee the velocity and the moving range of the mobiles to adjust sleeping time intelligent for decreasing the probability of generating the blind holes in some degree. Here we consider that the reliability of network communication is equal to the connection rate of mobile nodes.

\subsection{Sensing Coverage Percentage}

Next, we discuss the sensing coverage problem in DM-MAC (because sensing radius is equal to communication radius, the problem of sensing coverage is equal to communication coverage), in the simulation, we bring in 40 mobile nodes, the number of active nodes and coverage percentage in the network is shown in Figures $\mathbf{1 5}$ and 16. Obviously, as Figure 15 shows, when the Qos requirement improves (with the increase of $n, n$ is the number of awaken neighbor nodes), the required active neighbor nodes need to increase, so it leads to increase the active nodes in the network. In the Figure 16 we know that the network coverage of DM-MAC protocol and LDAS algorithm all achieve 100\% with increasing the number of neighbors required in QoS, but when the value of $n$ is small, the coverage of DM-MAC is obvious lager than LDAS algorithm. Because when bringing in

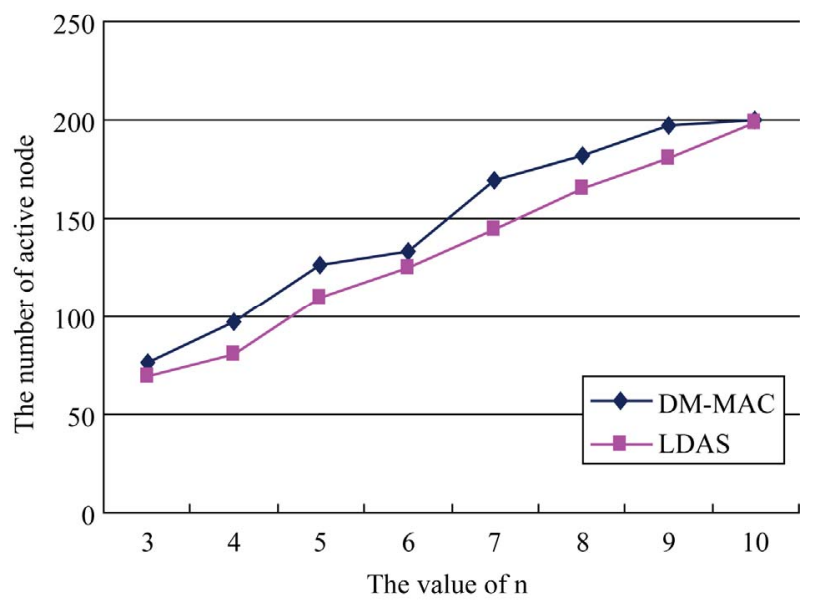

Figure 15. The number of active node. 


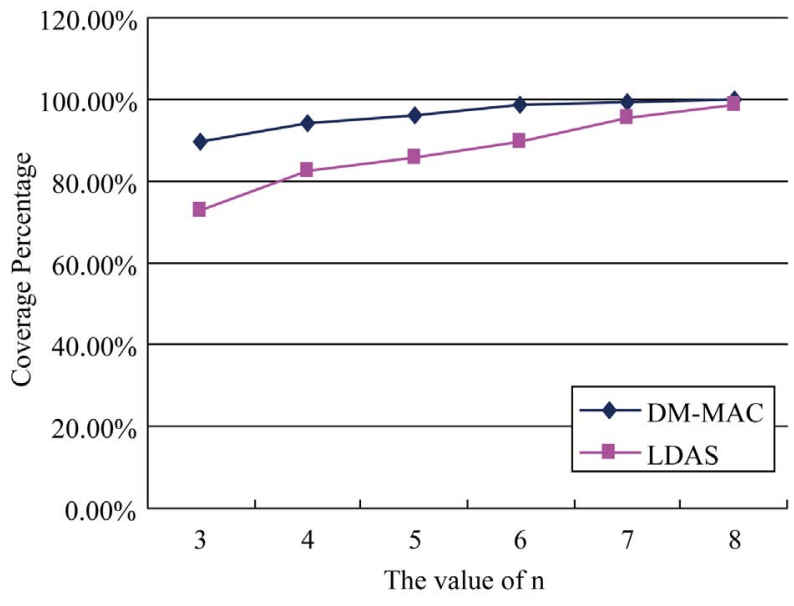

Figure 16. The network coverage degree.

mobile nodes, the scheduling mechanism of stationary nodes in DM-MAC continues changing (generally the value of ST becomes small), the number of sleeping nodes decreases, the coverage area of blind holes decreases and the probability of blind holes appearing decreases. But when the $n$ value increases, the number of neighbors also increases, and the probability of blind holes appearing decreases correspondingly. Though the simulation test, it is shown that when $n=8$, the average of network coverage in DM-MAC will achieve up to $99.80 \%$, and the average of network coverage in LDAS will reach $98.40 \%$. Therefore, when $n$ is relative large, the two kinds of algorithm will approximately realize the full network being covered.

\subsection{Energy Consumption}

At last, we discuss the problem of protocol energy consumption. LDAS algorithm only realizes the sleeping mechanism which doesn't propose a kind of reliable point-to-point communication mechanism, so comparing the energy consumption between LDAS and DM-MAC is not necessary. Here we give a comparison between EAR + SMACS protocol, SMACS + EAR and DM-MAC protocol; we set the occurrence frequency of discrete event is $0.6 \mathrm{time} / \mathrm{m}^{2} / \mathrm{sec}, n=3$. The result is shown in Figure 17.

From the above figure, we know that with the simulation time going, the decreasing of energy in EAR + SMACS is relatively faster, this is because EAR + SMACS uses the topology of cluster structure, the energy consumption is large in building cluster stage. Furthermore, in every period of time there will be a process of choosing cluster head in every cluster, and it applies TDMA technology to the algorithm which needs precise time synchronization. All of the above need to consume large energy. Meanwhile, when the occurrence frequency

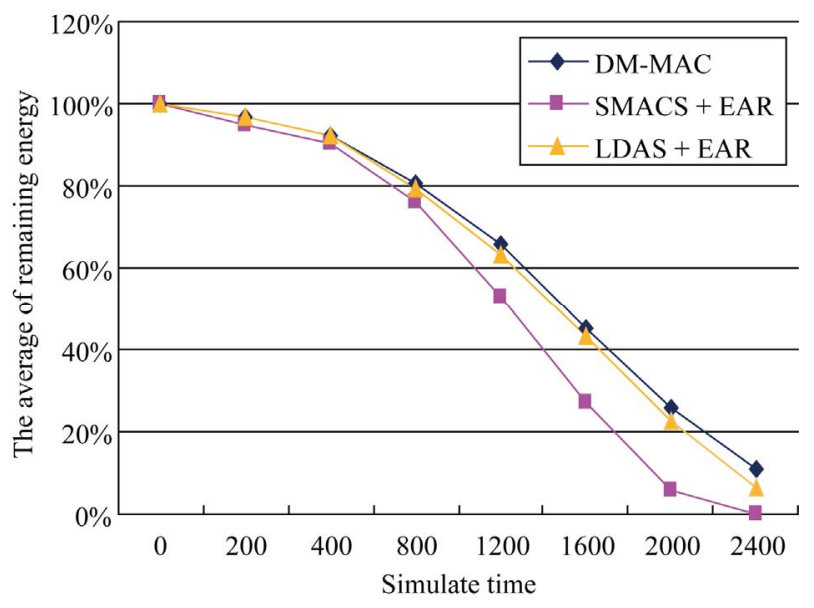

Figure 17. The average of node energy consumption.

of discrete event is low, the energy efficiency of EAR + SMACS is high, but when the frequency increases, the energy efficiency quickly decreases due to the nodes awakening frequently. On the other hand, the energy efficiency of LDAS + EAR and DM-MAC protocol are relative high, and the two protocols' energy consumption are almost the same. This is because the two MAC protocols is contention-based MAC protocol and they use period sleeping mechanism simply which save the energy greatly. In advance analyze, we find that DM-MAC do better than LDAS + EAR in energy efficiency, this is because that DM-MAC utilizes dynamic scheduling mechanism to adapt to energy saving, also, the most number of control packets in DM-MAC are sent by mobile nodes whose energy is considered to be limitless, it decrease the stationary nodes' energy consumption greatly. Meanwhile, the sleeping mechanism of DM-MAC and LDAS + EAR based on network coverage can basically realize the energy averaging of nodes in the network, thus prolongs the network life time.

\section{Conclusions}

DM-MAC is proposed under the condition of stationary nodes distributing randomly with a certain amount of mobile nodes existing in the network, it inherits the advantage of LDAS algorithm which needn't to know neighbors' geographical position information, dynamically adjusts the network density, has strong anti-interference ability on channel and collision, blind holes appears randomly and so on. Meanwhile, DM-MAC combines with the reliable point-to-point communication which is proposed in the EAR and improves the energy efficiency of stationary nodes through mobile nodes to undertake the most transmission tasks, furthermore, DM-MAC applies power control technology to enlarge the transmit power of mobile nodes so as to realize the 
reservation mechanism between mobile nodes and stationary nodes. In order to decrease the communication loss rate of mobile nodes and time delay, DM-MAC combines with the scheduling mechanism in MS-MAC, which could adjust the sleeping time intelligently.

Generally speaking, DM-MAC protocol is simple to realize. The energy consumption of every node is nearly the same. Moreover, the protocol can basically realize the reliable communication, so it could be applied to the real time positioning and tracking system. But DM-MAC protocol doesn't consider the problem of network connectivity, so there is a problem that the stationary nodes near the sink node will consume the energy much faster, thus will make the network paralysis in advance. Our future work is to solve this problem and analyze the impacts on different values of $T T, S T, B T, V T$ and $W_{\max }$ for the performance of network.

\section{Acknowledgment}

This work is supported by the Scientific Research Fund of Hebei Province Education Department (2008110) and Scientific \& Technological Research and Development Projects of Handan in Hebei province (0821103041-2).

\section{References}

[1] V. Rajendran, K. Obraczka and J. Garcia-Luna-Aceves, "Energy-Efficient, Collision-Free Medium Access Control for Wireless Sensor Networks," Proceedings of the ACM Sensor Systems 2003, Los Angeles, 2003, pp. 181192.

[2] A. El-Hoiydi and J.-D. Decotignie, "WiseMAC: An Ultra Low Power MAC Protocol for Multi-hop Wireless Sensor Networks," Algorithmic Aspects of Wireless Sensor Networks First International Workshop, Vol. 3121, 2004, pp. 18-31.

[3] K. Wu, Y. Gao, F. Li and Y. Xiao, "Lightweight Deployment-Aware Scheduling for Wireless Sensor Networks," ACM/Kluwer Mobile Networks and Applications (MONET) Special Issue on Energy Constraints and Lifetime Performance in Wireless Sensor Networks, Vol. 10, No. 6, 2005, pp. 837-852.

[4] K. Sourabi, J. Gao, V. Ailawadni and G. J. Pottie, "Protocols for Self-Organization of a Wireless Sensor Net- work," IEEE Personal Communications, Vol. 7, No. 5, 2000, pp. 16-27. doi:10.1109/98.878532

[5] H. Pham, and S. Jha, "An adaptive Mobility-Aware MAC Protocol for Sensor Networks (MS-MAC)," IEEE International Conference on Mobile Ad-hoc and Sensor Systems, 25-27 October 2004, pp. 558-560.

[6] W. Ye, J. Heidemann and D. Estrin, "Medium Access Control with Coordinated Adaptive Sleeping for Wireless Sensor Networks," IEEE/ACM Transactions on Networking, Vol. 12, No. 3, 2004, pp. 493-506. doi:10.1109/ TNET.2004.828953

[7] W. Ye, J. Heidemann and D. Estrin, "An Energy-Efficient MAC Protocol for Wireless Sensor Networks," INFOCOM 2002, pp. 1567-1576.

[8] B. Kusy, A. Ledeczi and X. Koutsoukos, "Tracking Mobile Nodes Using RF Doppler Shifts," ACM Sensor Systems, 2007, pp. 29-42.

[9] L. Wang and Y. Xiao, "A Survey of Energy-Efficient Scheduling Mechanisms in Sensor Networks," Mobile Networks and Applications, Vol. 11, No. 5, 2006, pp. 723-740. doi:10.1007/s11036-006-7798-5

[10] P. Raviraj, H. Sharif, M. Hempel and S. Ci, "MOBMAC-An Energy Efficient and Low Latency MAC for Mobile Wireless Sensor Networks,” 2005, pp. 370-375.

[11] T. V. Dam and K. Langendo, "An Adaptive Energy- Efficient MAC Protocol for Wireless Sensor Network," The First ACM Conference on Embedded Networked Sensor Systems, 2003, pp. 171-180.

[12] M. Ali, T. Suleman and Z. Uzmi, "MMAC: A Mobility-Adaptive, Collision-Free MAC Protocol for Wireless Sensor Networks," Proceedings of 24th IEEE Performance, Computing, and Communications Conference, 2005, pp. 401-407.

[13] D. Tian, N. D. Georganas, "A Coverage-Preserving Node Scheduling Scheme for Large Wireless Sensor Networks," Proceedings of the 1st ACM International Workshop on Wireless Sensor Networks and Applications, 2002, pp. 32-41. doi:10.1145/570738.570744

[14] A. L. Henrique, F. Daniel, L. dos Aldri, S. J. Marcos, and A. A. Ferreira, "A Taxonomy for Medium Access Control Protocols in Wireless Sensor Networks," Annales des Télécommunications, Vol. 60, No.1, 2005, pp. 944-969.

[15] H. Yousefi'zadeh, H. Jafarkhani and J. Kazemitabar, "A Study of Connectivity in MIMO Fading Ad-Hoc Networks," Journal of Communication and Networks, Vol. 11, 2009, pp. 47-56. 